
\title{
25 Research Square \\ Effects of zoledronic acid combined with metformin on bones of DB/DB mice
}

\section{Xingyao Yang}

Department of Orthopaedics, The First Affiliated Hospital of Chengdu Medical College, Chengdu, Sichuan Province

\section{Wen Zhao}

Department of Orthopaedics, The First Affiliated Hospital of Chengdu Medical College, Chengdu, Sichuan Province

\section{Wenjie Ma}

Department of Orthopaedics, The First Affiliated Hospital of Chengdu Medical College, Chengdu, Sichuan Province

\section{Xin Yin}

Department of Orthopaedics, The First Affiliated Hospital of Chengdu Medical College, Chengdu, Sichuan Province

\section{Yanshui Lin ( $\nabla 10580361 @ q q . c o m$ )}

Department of Orthopaedics, The First Affiliated Hospital of Chengdu Medical College, Chengdu, Sichuan Province

\section{Research Article}

Keywords: DB/DB mice, type 2 diabetes, osteoporosis, zoledronic acid, metformin

Posted Date: November 12th, 2020

DOl: https://doi.org/10.21203/rs.3.rs-103504/v1

License: (9) This work is licensed under a Creative Commons Attribution 4.0 International License. Read Full License 


\section{Abstract \\ Background}

Diabetic patients are prone to osteoporosis. Both zoledronic acid and metformin have certain antiosteoporotic effects. Here we explore the anti-osteoporotic effect of the combination of two drugs.

\section{Methods}

12-week-old DB/DB mice were divided into DB/DB group, zoledronic acid group,metformin group and zoledronic acid combined with metformin group, WT mice were treated as WT group alone. The mice were killed after ten weeks. Then using Micro-ct to scan the tibia and stain the contralateral lower limbs with HE.

\section{Results}

First, we find that the body weight of DB/DB mice treated with metforminre stable and their blood glucose reduce.Second, after HE staining, it is observed under light microscope that there are a large number of adipocytes, few bone trabeculae, few osteoblasts and osteoclasts in the bone marrow cavity in the DB/DB group compared with the WT group, while the number of bone trabeculae in the combined treatment group is higher than that in the zoledronic acid group or metformin group, and a large number of blood cells, blood vessels and adipocytes are found in the bone marrow cavity of the combined drug group compared with the zoledronic acid group. Last, the results of Micro-ct present that, comparing with the $D B / D B$ group and the metformin group,SMI is significantly different $(P<0.05)$;comparing with the $\mathrm{DB} / \mathrm{DB}$ group and the zoledronic acid group,Tb.N,Tb.Sp,Conn.D and $\mathrm{SMI}$ are significantly different $(\mathrm{P}<$ 0.05);BV/TV, Conn.D, SMI, BMD, Tb.N and Tb.Sp in the combination treatment group are significantly difference than those in the $D B / D B$ group $(P<0.05)$.

\section{Conclusion}

In term of the bone mass lose of DB/DB mice, the treatment of the zoledronic acid combined with metformin outperforms that using the zoledronic acid or metformin only.

\section{Background}

China has the largest number of people with diabetes in the world,it is expected that the number of people with type 2 diabetes will be reach 440 million by 2030[1]. Diabetes and osteoporosis, as two common chronic diseases, have shown an upward trend year by year with the intensification of social aging. The two chronic diseases not only have a huge economic burden on the society, but also bring misfortune to the patients' own mental and physical health. Diabetic patients have pathological changes such as 
decreased bone mass, increased bone fragility and damaged bone microstructure, which is called Diabetic Osteoporosis(DO).DO can significantly increase the risk of brittle fracture, which cause long-term severe pain and motor dysfunction, and improve the disability and mortality of patients[2,3]. At present, there is no specific drug for DO, and the effect of osteoporosis treatment after fracture is limited. Statistically, there are about $30 \%$ of osteoporotic fracture patients have secondary fractures within one year. Then for elderly patients, how to correctly choose anti-osteoporosis drugs and hypoglycemic drugs to reduce the risk of fracture is an urgent problem to be solved. Therefore, In order to explore the effect of the combination of two drugs on bone metabolism and obtain the results of drug effect after observation, this study uses Micro-CT to observe of bone mass changes in the tibial plateau of the tested mice after taking the zoledronic acid combined with metformin. Furthermore, this study provides a basic treatment for elderly patients with type 2 diabetes complicated with osteoporosis and reduce their risk and pain of fracture, which could improve patients' family happiness.

\section{Method}

Ethical approval

All procedures were approved by the Experimental Animal Care and Use Committee of the first affiliated Hospital of Chengdu Medical College and performed in accordance with the Guidelines for the Care and Use of Laboratory Animals (NIH publication No. 85-23, revised 1996).

Animal

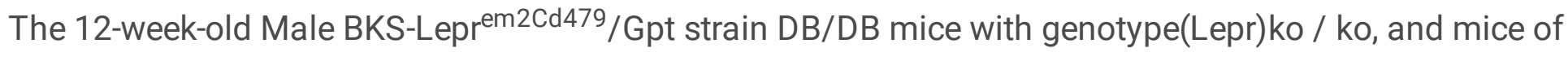
the same strain with genotype(Lepr)wt / wt are selected and included in the study(Purchased from Jiangsu GemPharmatech Co,Ltd ,China). First, we allocate 6 mice into WT group. Then, the left DB/DB mice are randomly divided into four groups:DB/DB group $(n=5)$, zoledronic acid group $(n=6)$, metformin group $(n=6)$ and combination group $(n=6)$, respectively.

Dosing and feeding

During the feeding period, All experimented mice are given enough pure water and food. The room temperature is between $20-25^{\circ} \mathrm{C}$, and the light and dark are irradiated alternately for $12 \mathrm{~h} / 12 \mathrm{~h}$. For dosing process, the WT group (WT) and the DB/DB group(DB/DB) are given equal saline daily. The zoledronic acid group $(\mathrm{ZOL})$ is injected by the $100 \mathrm{ug} / \mathrm{kgzoledronic}$ acid injection though intraperitoneal injection once,then given equal saline daily. ${ }^{[16]}$ The Metformin group(MET) are given gavage of $300 \mathrm{mg} / \mathrm{kg}$ metformin solution daily. As for combination treatment group(CMT), the experimented mice are intraperitoneally injected the $100 \mathrm{ug} / \mathrm{kg}$ zoledronic acid only once at the beginning of the experiment, then followed by gavage of $300 \mathrm{mg} / \mathrm{kg}$ metformin solution every day. The normal fodder are fed to the mice in five groups, then mice are fasted for 8 hours before the weekly measurement of the mice' blood glucose and body weight, in which the blood is collected through the tail vein and using blood glucose meter to test the blood glucose value of the mice.If is higher than the detected maximum value, then recorded as 
$33.3 \mathrm{mmol} / \mathrm{L}$.Feeding five groups mice for ten weeks and killed by cervical dislocation, then take out the lower limbs and fix with formaldehyde after removing soft tissue.

\section{Micro-ct}

The tibia of mice is put into Micro-CT(model: ZKKS-MCT-Sharp) for X-ray scanning. Data processing software: ZZKS-MicroCT4.1. The experimental samples is fixe in the fixator along the long axis, the energy and intensity is $70 \mathrm{kVP}$ and $100 \mu \mathrm{A} 7 \mathrm{~W}, 4$ frames superimposed exposure time $100 \mathrm{~ms}$, and the scanning time is $12 \mathrm{~min}$. After scanning, the bone tissue with $0.7 \mathrm{~mm}$ from the distal end of the growth plate and $1.5 \mathrm{~mm}$ in thickness is selected as the region of interest (ROI) of cancellous bone for threedimensional reconstruction, and the image information is extracted with the lowest threshold of 66 . The software(SCANCO Medical AG) is used for reconstruction and quantitative analysis. The parameters are as follows:connectivity density(Conn.D), structure model index(SMI), bone mineral density (BMD), bone volume/tissue volume(BV/TV), trabecular number(Tb.N), trabecular thickness (Tb.Th), trabecular separation(Tb.Sp).(Scanning services provided by Guangzhou Zhongke Kaisheng Medical Technology Co, Ltd,China)

HE staining

In order to take contralateral tibia for pathological examination, we fix contralateral tibia in $4 \%$ paraformaldehyde for 48 hours, and followed by gradient alcohol dehydration, paraffin embedding, section and HE staining, then make observations and take photographs under microscope.

Data analysis

The data of this study are analyzed by SPSS 25.0 statistical software, and the quantitative data are expressed as mean \pm standard deviation (SD); as for quantitative data, it is tested by normality test first, and then analyzed by one-way ANOVA, if the variance is homogeneity by Levene test, the LSD test is used between groups; if the variance is uneven, welch correction is used for ANOVA, and Dunnett's T3 multiple test is used between groups, statistically significant according to $P<0.05$.

\section{Results}

Body weight and blood sugar of mice

Table 1, Table 2, Figure1 and Figure 2 record the body weight and blood glucose of all mice during experiment period. As for the WT group, the body weight of mice increases slowly and their blood glucose remain stable. Although the body weight of the DB/DB group and the zoledronic acid group have the same tendency with the WT group, their blood glucose stays at a high level. However, the body weight of mice in the metformin group or the combined treatment group are slightly reducing and their blood glucose drops significantly.

HE staining 
Figure 3 and Figure 4 were magnified by 40 times and 200 times under ordinary light microscope. In WT group, there were more bone trabeculae and more blood cells in bone marrow cavity, while in DB/DB group, there were a large number of adipocytes in bone marrow cavity, few bone trabeculae, sparse and thin with fracture, and almost no osteoblasts and osteoclasts. In the zoledronic acid group, the bone trabecula was broken, the number was less, the structure was disordered, the gap was enlarged, including a large number of adipocytes and a small number of osteoblasts, while in the metformin group and the combination treatment group, there were more trabeculae, a large number of blood cells in the bone marrow cavity and few adipocytes, and a small number of osteoblasts and osteoclasts could be observed.

The results of Micro-ct

The table 3 presents the results based on SPSS analysis after the scanning of Micro-ct.It can be seen that Conn.D, SMI, BMD, Tb.N, Tb.Th and Tb.Sp in DB/DB group are significantly different from those in WT group $(P<0.05)$.Comparison of zoledronic acid group with DB/DB group, Conn.D, SMI, Tb.N, Tb.Sp are significantly different $(P<0.05)$;comparing with the metformin group and the DBDB group, only the SMI is different $(P<0.05)$; the combination treatment group compared with the DB / DB mouse group, BV / TV, Conn. D, SMI, BMD, Tb.N, Tb. Sp are significantly different $(P<0.05)$.Figure 5 is a bone reconstruction image of mice in each group after Micro-ct scan,we can see that the bone trabeculaes of DB / DB mice were significantly reduced, while that of CMT group was significantly increased.Figure 6 is a data analysis chart of mice after each group Micro-ct scan.

Table 1: The weight of mice(g)

\begin{tabular}{ccccccc}
\hline & $12 \mathrm{~W}$ & $14 \mathrm{~W}$ & $16 \mathrm{~W}$ & $18 \mathrm{~W}$ & $20 \mathrm{~W}$ & $22 \mathrm{~W}$ \\
\hline WT & $21.67 \pm 1.21$ & $22.50 \pm 0.55$ & $24.16 \pm 0.98$ & $25.67 \pm 0.82$ & $26.677 \pm 1.21$ & $28.00 \pm 0.80$ \\
& & & & & & \\
DB/DB & $42.00 \pm 2.55$ & $43.00 \pm 2.92$ & $44.20 \pm 2.17$ & $46.60 \pm 2.07$ & $48.80 \pm 2.39$ & $51.40 \pm 2.30$ \\
& & & & & & \\
ZOL & $42.17 \pm 2.99$ & $43.83 \pm 3.25$ & $46.17 \pm 3.37$ & $48.67 \pm 3.98$ & $50.67 \pm 3.50$ & $53.00 \pm 3.58$ \\
& & & & & $38.33 \pm 2.07$ & $37.83 \pm 2.32$ \\
MET & $41.83 \pm 2.84$ & $41.83 \pm 2.64$ & $40.50 \pm 2.35$ & $39.17 \pm 2.40$ & $38.00 \pm 5.17$ \\
& & & & & & \\
\hline
\end{tabular}

Tab2 Blood glucose of mice (mmol/L)

\begin{tabular}{ccccccc}
\hline & $12 \mathrm{~W}$ & $14 \mathrm{~W}$ & $16 \mathrm{~W}$ & $18 \mathrm{~W}$ & $20 \mathrm{~W}$ & $22 \mathrm{~W}$ \\
\hline $\mathrm{WT}$ & $8.98 \pm 0.98$ & $9.45 \pm 1.02$ & $9.43 \pm 0.65$ & $9.17 \pm 1.11$ & $9.15 \pm 1.03$ & $9.32 \pm 0.80$ \\
\hline DB/DB & $30.78 \pm 2.37$ & $32.40 \pm 1.65$ & $31.24 \pm 1.87$ & $32.16 \pm 1.39$ & $32.36 \pm 0.89$ & $32.60 \pm 0.62$ \\
\hline ZOL & $28.98 \pm 3.59$ & $31.01 \pm 2.58$ & $30.55 \pm 1.72$ & $30.70 \pm 1.71$ & $31.23 \pm 2.08$ & $30.25 \pm 2.48$ \\
\hline MET & $32.55 \pm 0.99$ & $28.22 \pm 3.08$ & $23.83 \pm 2.97$ & $21.53 \pm 0.92$ & $18.83 \pm 3.47$ & $16.25 \pm 4.18$ \\
\hline CMT & $30.42 \pm 6.04$ & $27.93 \pm 4.00$ & $25.35 \pm 3.48$ & $21.93 \pm 4.74$ & $17.50 \pm 5.90$ & $14.82 \pm 7.26$ \\
\hline
\end{tabular}


Tab3 Data after micro-ct scan

\begin{tabular}{|c|c|c|c|c|c|c|c|}
\hline & BV/TV(\%) & $\operatorname{Tb} . \operatorname{Th}(\mathrm{mm})$ & $\mathrm{Tb} . \mathrm{N}(1 / \mathrm{mm})$ & $\mathrm{Tb} . \mathrm{Sp}(\mathrm{mm})$ & Conn.Dn(g/cmI) & $\mathrm{BMD}(\mathrm{g} / \mathrm{cm}])$ & SMI \\
\hline WT & $3.61 \pm 1.06$ & $0.05 \pm 0.01$ & $0.86 \pm 0.21$ & $0.38 \pm 0.05$ & $72.35 \pm 10.98$ & $0.24 \pm 0.04$ & $2.33 \pm 0.20$ \\
\hline $\mathrm{DB} / \mathrm{DB}$ & $1.85 \pm 0.33$ & $0.05 \pm 0.03^{*}$ & $0.35 \pm 0.07 *$ & $0.62 \pm 0.04^{*}$ & $27.09 \pm 7.76^{*}$ & $0.15 \pm 0.01^{*}$ & $3.03 \pm 0.19^{*}$ \\
\hline $\mathrm{ZOL}$ & $2.43 \pm 0.34$ & $0.05 \pm 0.01 *$ & $0.54 \pm 0.06{ }^{* \dagger}$ & $0.49 \pm 0.05^{* \dagger}$ & $63.85 \pm 14.57^{\dagger}$ & $0.18 \pm 0.02$ & $2.49 \pm 0.15^{\dagger}$ \\
\hline MET & $2.02 \pm 0.34$ & $0.05 \pm 0.01$ & $0.41 \pm 0.07^{*}$ & $0.58 \pm 0.07^{* \ddagger}$ & $40.03 \pm 11.53^{* \ddagger}$ & $0.16 \pm 0.02 *$ & $2.66 \pm 0.27^{\dagger}$ \\
\hline CMT & $2.80 \pm 0.48^{\dagger}$ & $0.05 \pm 0.01$ & $0.67 \pm 0.06^{*+\S}$ & $0.47 \pm 0.03^{* \dagger \S}$ & $69.43 \pm 10.43^{\dagger \S}$ & $0.20 \pm 0.02^{\dagger}$ & $2.20 \pm 0.10^{\dagger \neq \S}$ \\
\hline
\end{tabular}

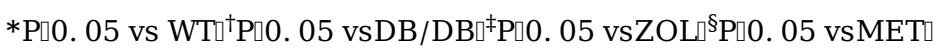

\section{Discussion}

According to the previous experiments, we find that DB/DB mice showed physiological symptoms such as hyperglycemia, hyperlipidemia, obesity, overeating and polyuria from 4 weeks after birth, which are widely used in animal studies of type 2 diabetes[17]. Related studies find that DB/DB mice secondary to decreased bone mass[18], osteocalcin level and bone cortex strength, ${ }^{[19]}$ so we consider it as an animal model of type 2 diabetes complicated with osteoporosis.

Many scholars have carried out clinical and basic experimental studies on metformin against osteoporosis, and there are still many disputes. Metformin are used internationally as first-line oral medication for patients with type 2 diabetes in clinical work[4]. Number of related studies have shown that metformin can effectively reduce the risk of diabetic fracture[5]. However, some relevant studies find that the usage of metformin drugs is not significantly associated with the risk of vertebral fracture and it cannot promote bone formation, as well as, cannot delay the process of osteoporosis[6,10]. In the study of cellular level, it is found that Bone mesenchymal stem cells(BMSCs) can differentiate into chondrocytes, osteoblasts and osteoclasts according to different environments. When osteoporosis occurs, bone mass will decrease and fat in bone marrow will increase[20]. Metformin can promote the proliferation and differentiation of bone-differentiated BMSCs in vitro, but inhibit the differentiation of lipid-differentiated BMSCs in vitro[21]. Metformin can also promote the differentiation and mineralization of osteoblasts[6]. In the study of animal model, it was found that metformin could prevent bone loss in ovariectomized(OVX) rats[7]. increase the number of bone trabeculae and inhibit the growth of osteoclasts. After oral administration of metformin, the increase of osteoclasts was significantly decreased[9]. At the molecular level, metformin can promote the expression of osteogenesis-related genes RUNX2 and ALP, inhibit the expression of adipogenesis-related genes PPARY and C/EBPa [7],and inhibit osteoclast differentiation in Raw264.7 macrophages induced by nuclear factor kB ligand(RANKL)[8]. 
From the experimental results in Figure 1 and Figure 2, we can see that after gavage of metformin, the blood glucose decrease and their body weight decrease slightly in the group of the DB/DB mice.Metformin is also effective in preventing people at high risk of type 2 diabetes. More than $60 \%$ of this effect is attributed to metformin's ability to lose weight continuously[22]. For patients with type 2 diabetes and normal people, metformin also has a certain weight loss effect, but the mechanism of metformin weight loss is not very clear, which may act on the central nervous system and reduce appetite by weakening hypothalamic AMPK activity[23].

The hypoglycemic mechanisms of metformin include reducing liver glucose production, enhancing peripheral insulin sensitivity and blocking gastrointestinal glucose absorption[24]. According to our results, the reason why the osteoblasts and osteoclasts are rarely found in the sections of DB/DB mice after $\mathrm{HE}$ staining is the environment of high glucose and hyperosmotic was not conducive to their growth[25,26]. Meanwhile, we stain with HE after adding metformin and find that the number of adipocytes in the medullary cavity decrease, blood vessels increase and hematopoietic cells increase, which may also be due to the weight loss and lipid lowering effect of metformin, which led to the decrease of adipocytes[27,28]. Subsequently,it has been made clear that microangiopathy is one of the main complications of diabetes, and blood vessels are the source of nutrients for bone tissue, so vascular injury will affect bone mass and bone structure to some extent[29]. Metformin can repair blood vessels by reducing inflammatory reaction and protecting vascular endothelial cells $[11,30]$. We also observe the increase of blood cells, which may be due to the increase of blood vessels, or because metformin itself has the function of promoting the proliferation of blood cells, which needs to be further explored to put forward ideas for further experiments;in addition, there are still few osteoblasts and osteoclasts stained by $\mathrm{HE}$ after the addition of metformin,Although in vitro experiments have proved that metformin can promote the proliferation and differentiation of bone-differentiated BMSCs, and inhibit the differentiation of lipid-differentiated BMSCs in vitro, it can also promote the differentiation and mineralization of osteoblasts. ${ }^{[6]}$ But according to the results of this experiment, it is not enough to inhibit the negative effects of high glucose and hypertonic environment on osteoblasts.

Zoledronic acid is the third generation bisphosphonate preparation for intravenous use. At present, zoledronic acid is mainly used in the treatment of Paget's disease, hypercalcemia and multiple myeloma, as well as bone loss caused by androgen deficiency with prostate cancer and osteolytic bone metastasis caused by a variety of tumors. In 2007, the US Food and Drug Administration approve zoledronic acid for the treatment of osteoporosis in postmenopausal women as a new choice for patients with osteoporosis and has been shown to prevent bone loss and increase bone mineral density in diabetic animals[12].Zoledronate bisphosphonate has been shown to promote the proliferation and osteogenic differentiation of BMSCs and increase the expression of OCN,RANKL,OPG and type II collagen[13].Other studies shows that short-term bisphosphonate treatment can prevent bone loss caused by diabetes, while long-term treatment can increase bone mineral density but inhibit bone formation in mice[14],while zoledronic acid inhibits osteoclast migration, differentiation and bone resorption through the AMPK pathway[15]. As an effective anti-osteoporosis drug,by Micro-ct scanning, we find that the bone of mice 
treated with zoledronic acid is significantly better than that of DB/DB mice. After adding metformin, DB/DB mice show advantages in the number of bone trabeculae and SMI, which indicated that metformin is superior to zoledronic acid alone in anti-bone mass loss and anti-osteoporosis. However,in this experiment,the advantages of using metformin alone are not obvious. Although the increase of blood vessels and trabeculae and the decrease of adipocytes can be seen from HE staining, only the SMI of Micro-ct data is different.Maybe the sample size is small,the difference of other bone microstructure indexes is not obvious, and there is statistical bias.

In this experiment, DB/DB mice are used as the model of type 2 diabetic osteoporosis to observe the effect of zoledronic acid combined with metformin on the bones of DB/DB mice.The bone condition of DB/DB mice treated with zoledronic acid was significantly better than that of mice without zoledronic acid. The blood glucose and body weight of DB/DB mice treated with metformin are lower than those of untreated DB/DB mice,but Micro-ct datas shows that metformin is not effective against bone loss. After $\mathrm{HE}$ staining of tibia of the combination treatment group,fusiform osteoblasts are observed on the periosteal surface, and there are a large number of blood cells, more blood vessels and fewer adipocytes in the medullary cavity.At the same time, comparing with DB/DB mice treated with zoledronic acid alone or metformin, the number of bone trabeculae increased and SMI decrease, and the difference of SMI also indicates the difference of osteoporosis. In conclusion, zoledronic acid combined with metformin can reduce bone mass loss in DB/DB mice to some extent, and is superior to zoledronic acid or metformin alone in anti-osteoporosis.

\section{Abbreviations}

DO:Diabetic osteoporosis;the WT:the WT group;DB/DB:the DB/DB group;ZOL:the zoledronic acid group;MET:the Metformin group;CMT:the combination treatment group;ROI:region of interest;Conn.D:connectivity density;SMl:structure model index;BMD:bone mineral density; BV/TV:bone volume/tissue volume;Tb.N:trabecular number;Tb.Th:trabecular thickness; Tb.Sp:trabecular separation

\section{Declarations}

Acknowledgments

Not applicable.

Authors' contributions

All authors were responsible for experimental design, animal feeding, and data processing.LYS and YXY were responsible for experimental design,HE stain of mice and article writing.ZW and MWJ completed feed and HE stain of mice,Data statistics and analysis are handled by YX.

Funding 
The funding comes from Science and Technology Project of China's Sichuan Provincial Health Commission(approval number 20PJ158) and the scientific research project of the First Affiliated Hospital of Chengdu Medical College (approval number CYFY2019YB01). All authors have stated that there is no conflict of interest.

Availability of data and materials

The datasets used and/or analyzed during the current study are available from the corresponding author upon reasonable request.

Ethics approval

All procedures were approved by the Experimental Animal Care and Use Committee of the first affiliated Hospital of Chengdu Medical College and performed in accordance with the Guidelines for the Care and Use of Laboratory Animals (NIH publication No. 85-23, revised 1996).

Consent for publication

Not applicable.

Competing interests

The authors declare that they have no competing interests.

Author details

${ }^{1}$ Department of Orthopaedics, The First Affiliated Hospital of Chengdu Medical College, Chengdu, Sichuan Province, 610500,China

\section{References}

[1] hang Wei-Lin,Meng Hong-Zheng, Yang Rui-Fei, Yang Mao-Wei, Sun Guang-Hong, Liu Jun-Hua, et al. (2016). Melatonin suppresses autophagy in type 2 diabetic osteoporosis. Oncotarget, 7(32), 5217952194. doi:10.18632/oncotarget.10538.

[2]Vestergaard P.(2007). Discrepancies in bone mineral density and fracture risk in patients with type 1 and type 2 diabetes-a meta-analysis. Osteoporos Int, 18(4), 427-44. doi:10.1007/s00198-006-0253-4.

[3]Räkel A, Sheehy O囚Rahme E囚LeLorier J囚et al. Osteoporosis among patients with type 1 and type 2 diabetes.Diabetes \& Metabolism,34(3), 193-205.doi:10.1016/j.diabet.2007.10.008.

[4]Inzucchi Silvio E,Bergenstal Richard M,Buse John B,Diamant Michaela『Ferrannini Ele『Nauck Michael et al. Management of Hyperglycemia in Type 2 Diabetes: A Patient-Centered Approach: Position 
Statement of the American Diabetes Association (ADA) and the European Association for the Study of Diabetes (EASD). Diabetes Spectrum, 2012. 25(3): p. 154-171.doi:10.2337/diaspect.25.3.154.

[5]Zinman Bernard,Haffner Steven M,Herman William H,Holman Rury R,Lachin John M,Kravitz Barbara G,et al.Effect of rosiglitazone, metformin, and glyburide on bone biomarkers in patients with type 2 diabetes. The Journal of Clinical Endocrinology \& Metabolism, 2010. 95(1): p. 134-

142.doi:10.1210/jc.2009-0572.

[6]Cortizo Ana M,Sedlinsky Claudia,McCarthy Antonio D,Blanco Alcira,Schurman León,et al. Osteogenic actions of the anti-diabetic drug metformin on osteoblasts in culture.European journal of pharmacology, 2006. 536(1-2): p. 38-46.doi:10.1016/j.ejphar.2006.02.030

[7]Gao Ying,Li Yunfeng,Xue Jing,Jia Yongqian,Hu Jing.Effect of the anti-diabetic drug metformin on bone mass in ovariectomized rats. European journal of pharmacology, 2010. 635(1-3): p. 231236.doi:10.1016/j.ejphar.2010.02.051.

[8]Lee Young-Sun,Kim Yang-Soon,Lee Sun-Young,Kim Geun-Hyang,Kim Beom-Jun,Lee Seung-Hun,et al.AMP kinase acts as a negative regulator of RANKL in the differentiation of osteoclasts. Bone, 47(5), 926-937. doi:10.1016/j.bone.2010.08.001.

[9]Mai Qi-guang,Zhang Zhong-min,Xu Song,Lu Ming,Zhou Rong-ping,Zhao Li,et al.Metformin stimulates osteoprotegerin and reduces RANKL expression in osteoblasts and ovariectomized rats. Journal of Cellular Biochemistry, 112(10), 2902-2909.doi:10.1002/jcb.23206.

[10]Kanazawa Ippei,Yamaguchi Toru,Yamamoto Masahiro,Sugimoto Toshitsugu.Relationship between treatments with insulin and oral hypoglycemic agents versus the presence of vertebral fractures in type 2 diabetes mellitus. J Bone Miner Metab 28,554-560 (2010). doi:10.1007/s00774-010-0160-9.

[11]Gnanapragasam Arunachalam, Arun Prasath Lakshmanan, Samson Mathews Samuel,Chris R. Triggle,Hong Ding. Molecular Interplay between microRNA-34a and Sirtuin1 in Hyperglycemia-Mediated Impaired Angiogenesis in Endothelial Cells: Effects of Metformin. Journal of Pharmacology and Experimental Therapeutics, 2016. 356(2): p. 314-323.doi:10.1124/jpet.115.226894.

[12]Duarte Poliana Mendes,de Vasconcelos Gurgel, Bruno César,Sallum Antonio Wilson,Nogueira Filho Getúlio Rocha,Sallum Enilson Antonio,Nociti Jr Francisco Humberto.Alendronate therapy may be effective in the prevention of bone loss around titanium implants inserted in estrogen-deficient rats. Journal of periodontology, 2005. 76(1): p. 107-114.doi:10.1902/jop.2005.76.1.107.

[13]Gong Xue,Yu Wanlu,Zhao Hang,Su Jiansheng,Sheng Qing.Skeletal Site-specific Effects of Zoledronate on in vivo Bone Remodeling and in vitro BMSCs Osteogenic Activity. Sci Rep 7, 36129 (2017). doi:10.1038/srep36129. 
[14]Coe L M,Tekalur S A,Shu Y,Baumann M J, McCabe L R.Bisphosphonate treatment of type I diabetic mice prevents early bone loss but accentuates suppression of bone formation. J. Cell. Physiol, 2015. 230(8): p. 1944-1953. doi:10.1002/jcp.24929.

[15]Dong W, Qi M,Wang Y,Feng X, Liu H.Zoledronate and high glucose levels influence osteoclast differentiation and bone absorption via the AMPK pathway. Biochemical and biophysical research communications, 2018. 505(4): p. 1195-1202.doi.:10.1016/j.bbrc.2018.10.059.

[16]Chen T,Berenson J,Vescio R,Swift R,Gilchick A,Goodin S, et al.(2002), Pharmacokinetics and Pharmacodynamics of Zoledronic Acid in Cancer Patients with Bone Metastases. The Journal of Clinical Pharmacology, 42: 1228-1236. doi:10.1177/009127002762491316.

[17]Garris David R.(2004). Estrogenic stimulation of ovarian follicular maturation in diabetes (db/db) mutant mice: restoration of euglycemia prevents hyperlipidemic cytoatrophy. Cell Tissue Res., 318(2), 365-73. doi:10.1007/s00441-004-0967-6.

[18] Hosokawa T.Altered bone metabolism in db/db mice. Nihon Ronen Igakkai zasshi. Japanese journal of geriatrics, 1992. 29(7-8): p. 540-548.doi:10.3143/geriatrics.29.540.

[19]Williams G A,Callon K E,Watson M,Costa J L,Ding Y, Dickinson M.(2011),Skeletal phenotype of the leptin receptor-deficient db/db mouse. J Bone Miner Res, 26: 1698-1709. doi:10.1002/jbmr.367.

[20]Benayahu D,Shur I,Ben-Eliyahu S. (2000), Hormonal changes affect the bone and bone marrow cells in a rat model. J. Cell. Biochem., 79: 407-415. doi:10.1002/1097-4644(20001201)79:3<407::AIDJCB60>3.0.C0;2-8.

[21]Gao Y,Xue J,Li X,Jia Y,Hu J.(2008),Metformin regulates osteoblast and adipocyte differentiation of rat mesenchymal stem cells. Journal of Pharmacy and Pharmacology, 60: 1695-1700. doi:10.1211/jpp.60.12.0017.

[22]John M Lachin, Costas A Christophi, Sharon L Edelstein, David A Ehrmann,Richard F Hamman, Steven E Kahn, et al.Factors Associated With Diabetes Onset During Metformin Versus Placebo Therapy in the Diabetes Prevention Program.Diabetes Apr 2007, 56 (4) 1153-1159; DOI: 10.2337/db06-0918.

[23]Malin Steven K,Kashyap Sangeeta R.(2014).Effects of metformin on weight loss: potential mechanisms.Curr Opin Endocrinol Diabetes Obes, 21(5), 323-9. doi:10.1097/MED.0000000000000095.

[24]Viollet Benoit,Guigas Bruno,Sanz Garcia Nieves,Leclerc Jocelyne,Foretz Marc, Andreelli Fabrizio. (2012). Cellular and molecular mechanisms of metformin: an overview. Clin. Sci., 122(6), 253-70. doi:10.1042/CS20110386.

[25]Rathinavelu Selvalakshmi,Guidry-Elizondo Crissy,Banu Jameela.(2018). Molecular Modulation of Osteoblasts and Osteoclasts in Type 2 Diabetes. J Diabetes Res, 2018(undefined), 6354787. doi:10.1155/2018/6354787. 
[26]Cai Zhen-Yu,Yang Bo,Shi Ying-Xu,Zhang Wei-Lin,Liu Fei,Zhao Wei,et al.(2018).High glucose downregulates the effects of autophagy on osteoclastogenesis via the AMPK/mTOR/ULK1 pathway. Biochem. Biophys. Res. Commun., 503(2), 428-435. doi:10.1016/j.bbrc.2018.04.052.

[27]Zhang Fan,Xiang Hao,Fan Yunzhou,Ganchuluun Tsend-Ayush,Kong Wenhua, Ouyang Qian, et al. (2013).The effects of sulfonylureas plus metformin on lipids, blood pressure, and adverse events in type 2 diabetes: a meta-analysis of randomized controlled trials. Endocrine, 44(3), 648-58.

doi:10.1007/s12020-013-9970-6.

[28]Morley Lara C,Tang Thomas,Yasmin Ephia,Norman Robert J,Balen Adam H.(2017).Insulin-sensitising drugs (metformin, rosiglitazone, pioglitazone, D-chiro-inositol) for women with polycystic ovary syndrome, oligo amenorrhoea and subfertility. Cochrane Database Syst Rev, 11(undefined), CD003053. doi:10.1002/14651858.CD003053.pub6.

[29]Spinetti Gaia,Cordella Daniela,Fortunato Orazio,Sangalli Elena,Losa Sergio, Gotti Ambra, et al. (2013).Global remodeling of the vascular stem cell niche in bone marrow of diabetic patients: implication of the microRNA-155/FOXO3a signaling pathway. Circ. Res., 112(3), 510-22.

doi:10.1161/CIRCRESAHA.112.300598.

[30]Isoda Kikuo,Young James L,Zirlik Andreas,MacFarlane Lindsey A,Tsuboi Naotake,Gerdes Norbert,et al. (2006).Metformin inhibits proinflammatory responses and nuclear factor-kappaB in human vascular wall cells. Arterioscler. Thromb. Vasc. Biol., 26(3), 611-7. doi:10.1161/01.ATV.0000201938.78044.75.

\section{Figures}

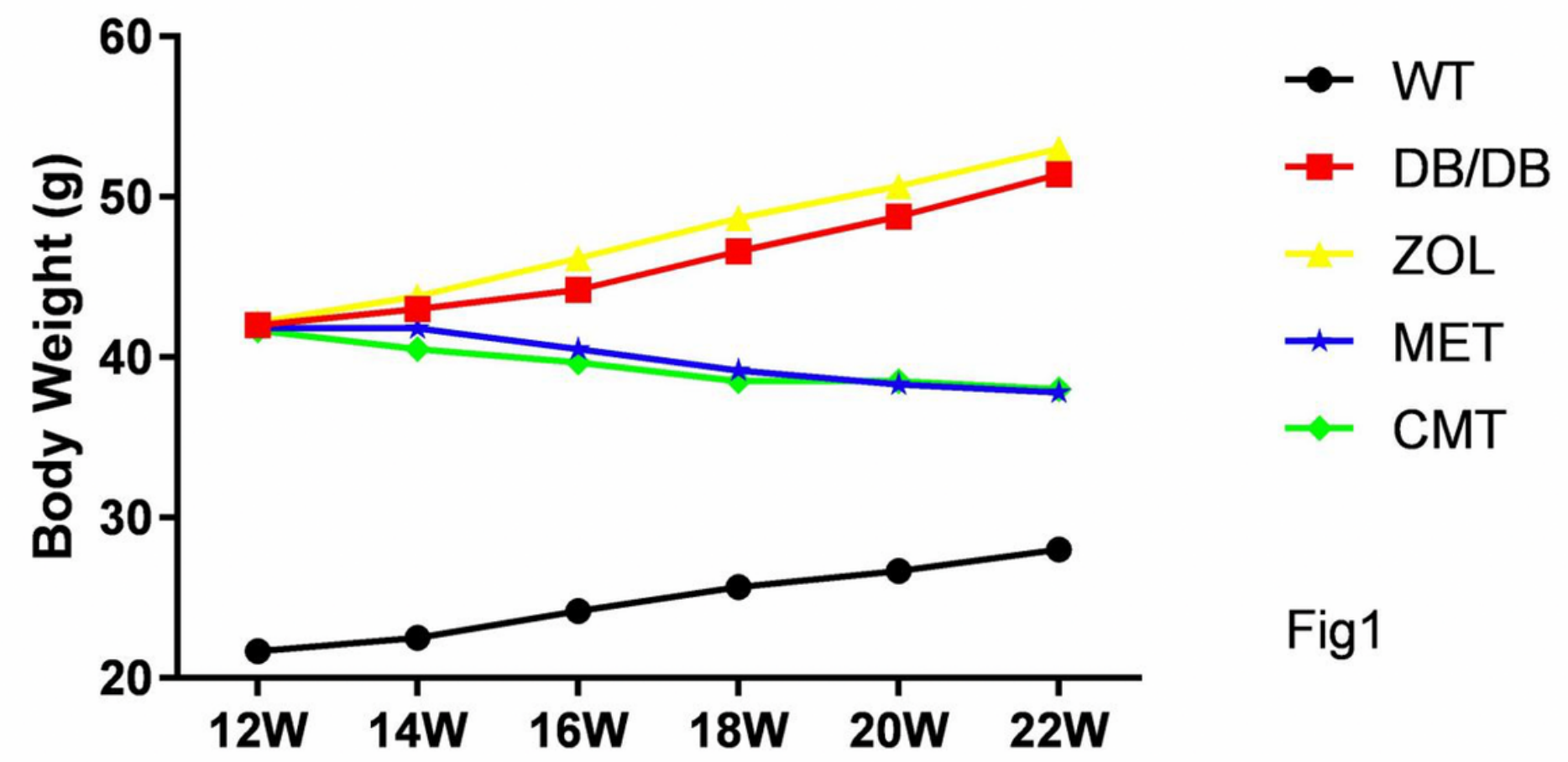


Figure 1

The weight of mice

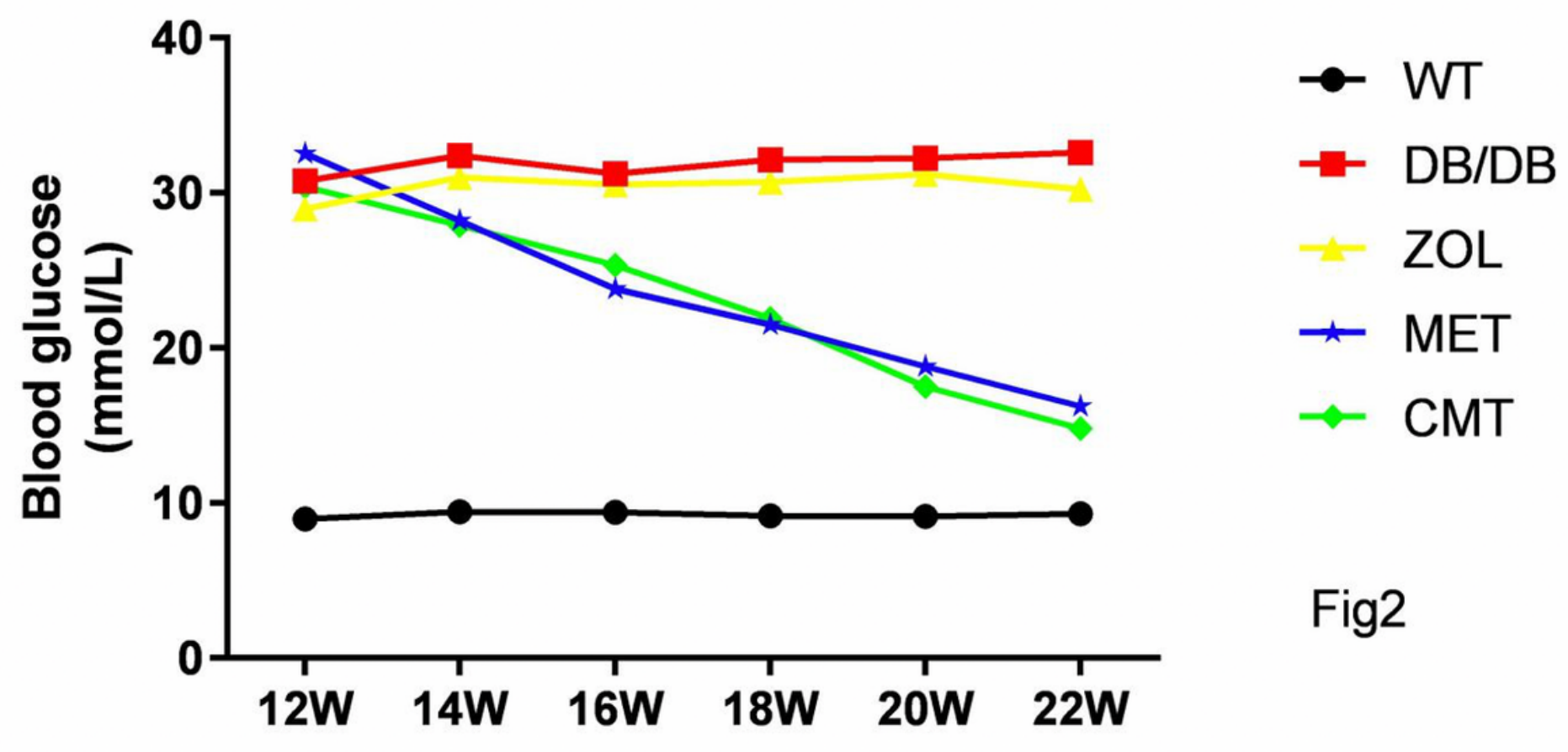

Figure 2

Blood glucose of mice 

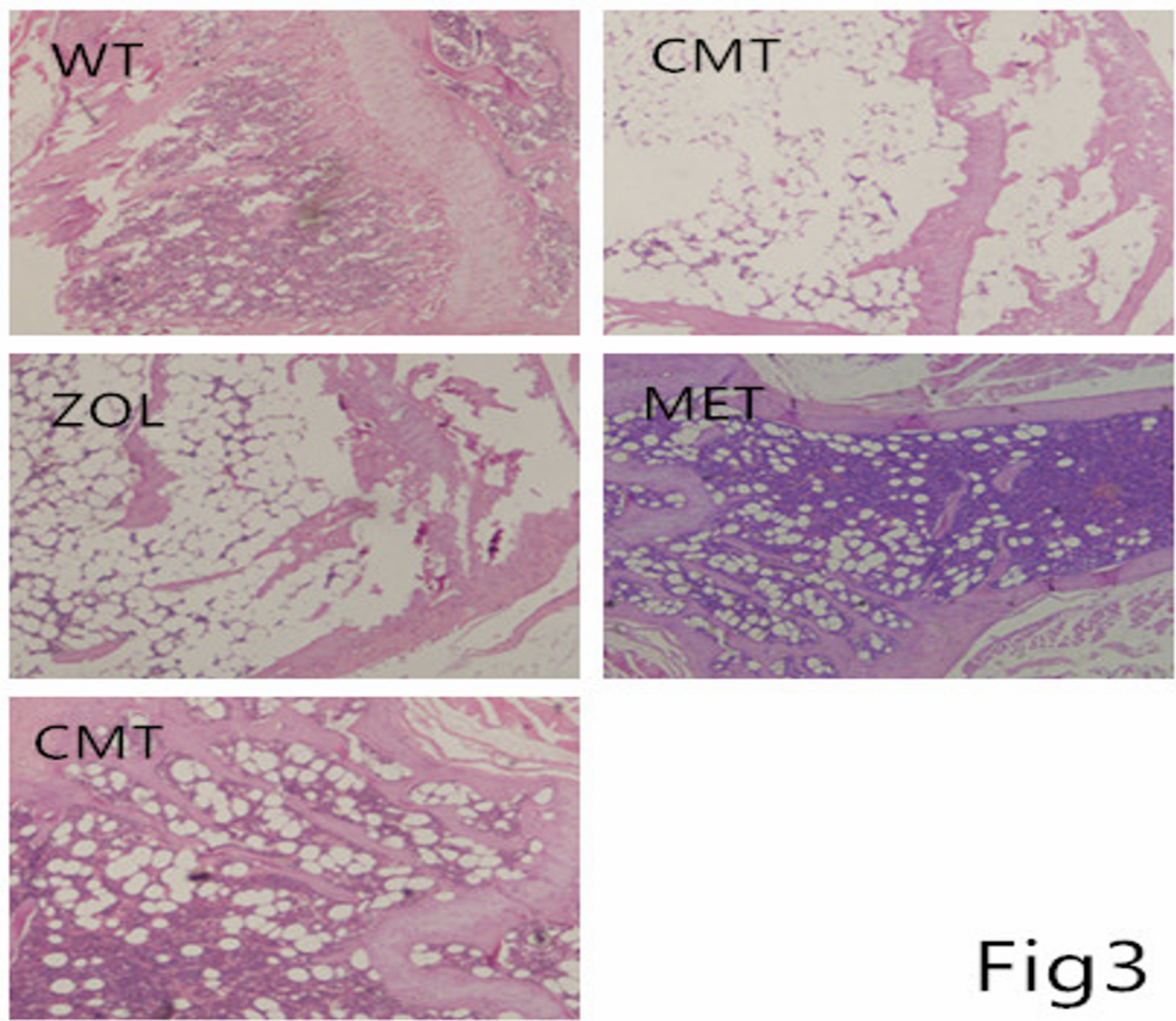

Fig3

Figure 3

Observation under 40 times ordinary light microscope of HE staining. 


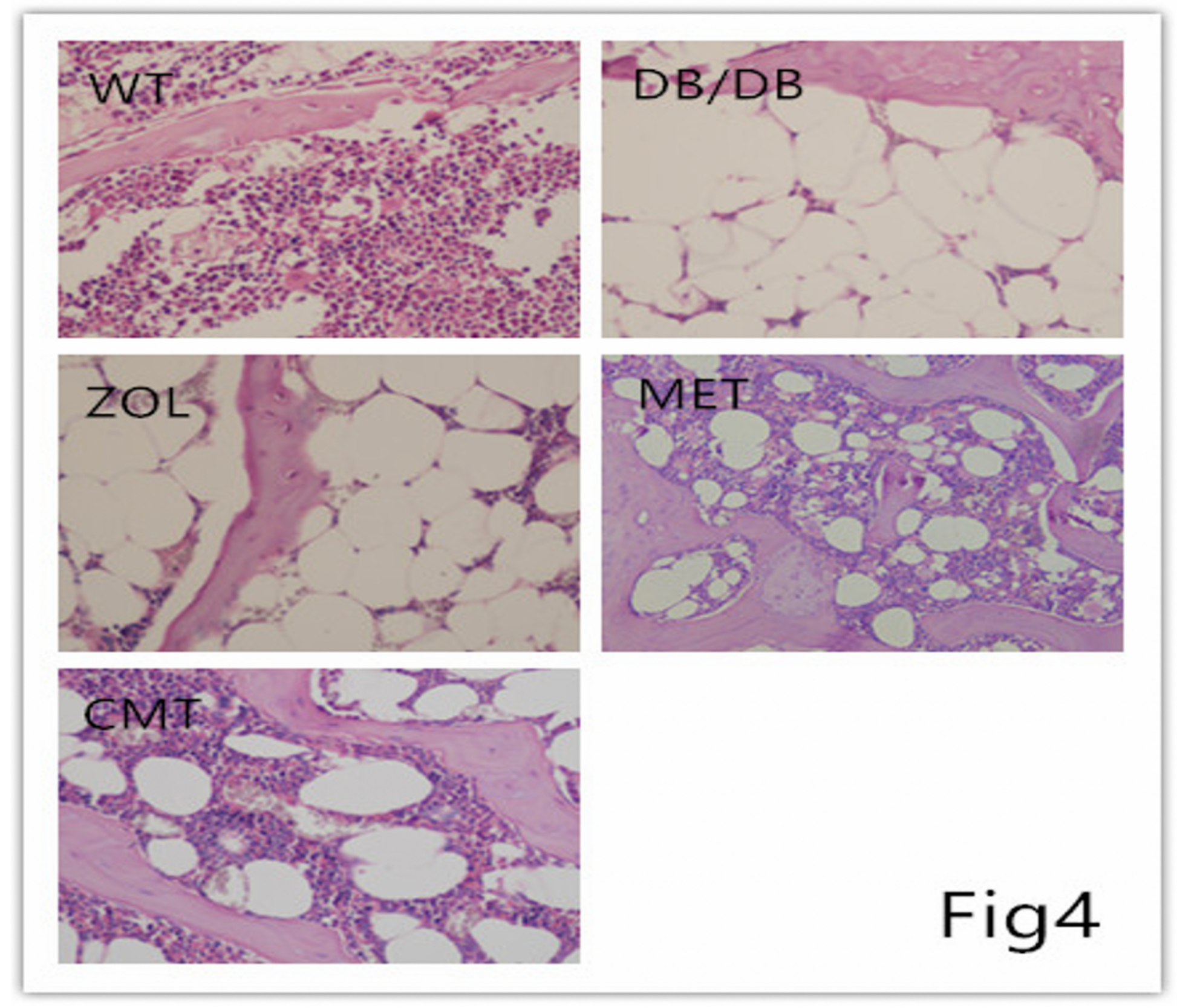

Figure 4

Observation under 200 times ordinary light microscope of HE staining. 


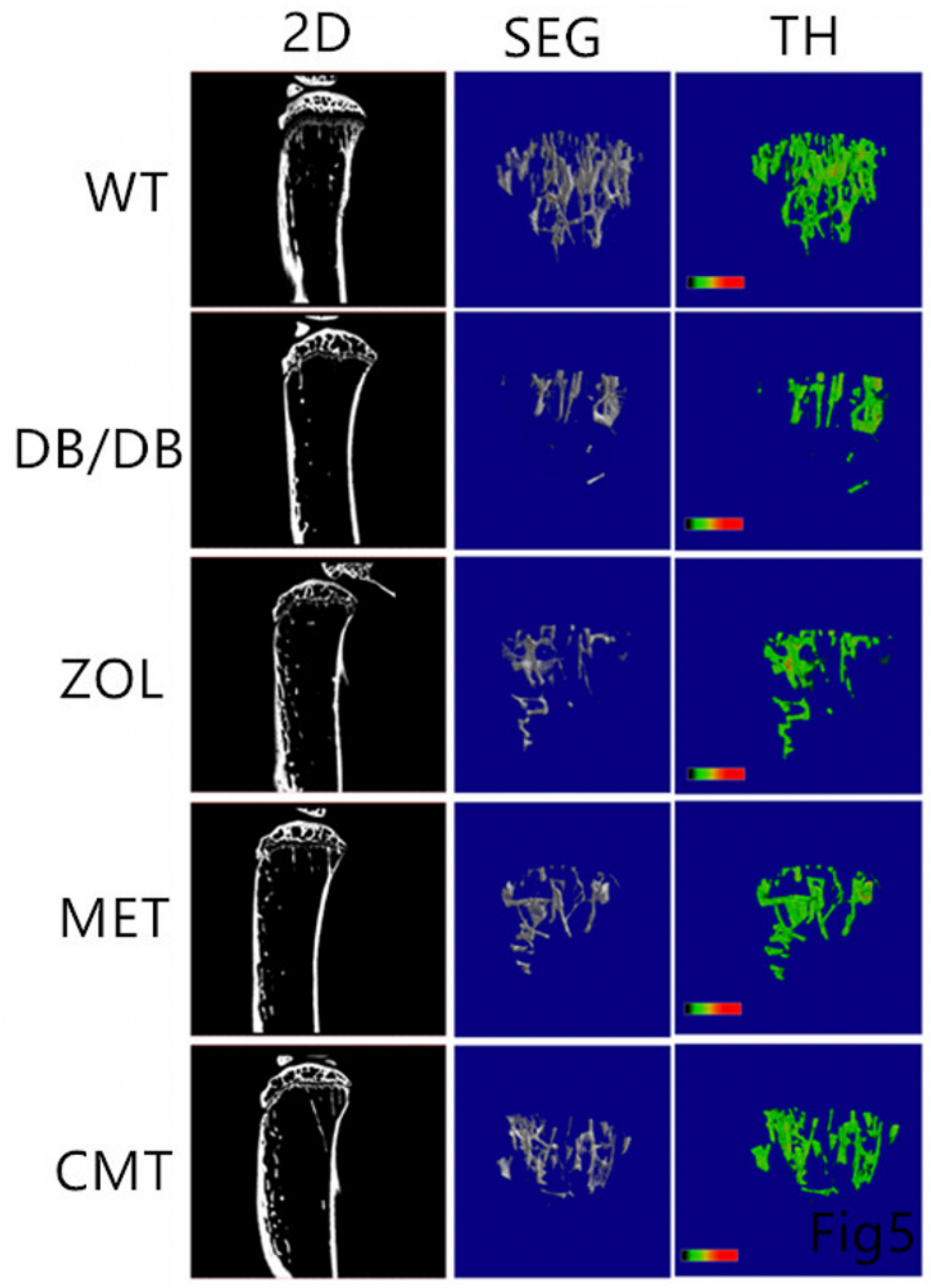

Figure 5

In the picture, two-dimensional map(2D image), shows the number and shape of bone trabeculae in bone marrow cavity.Segmented (SEG)reflects the number of bone trabeculae.Trabecular thickness(TH) map reflects the distribution of bone trabeculae, color from green to red, reflecting bone trabeculae from thin to thick. 

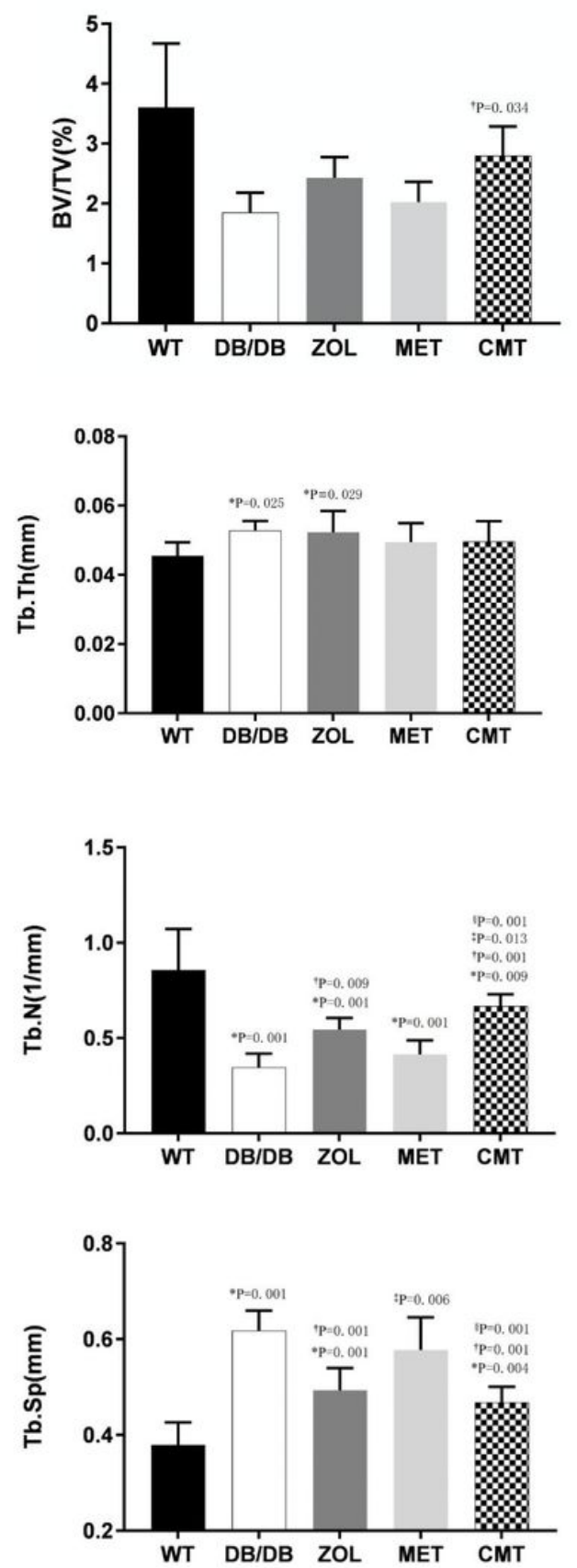

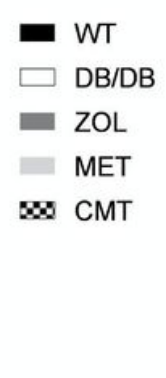

- WT

$\square$ DB/DB

- ZOL

MET

$\infty$ CMT

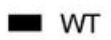

$\square$ DB/DB

- ZOL

MET

$\infty$ CMT

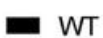

$\square$ DB/DB

- ZOL

- MET

$\infty$ CMT
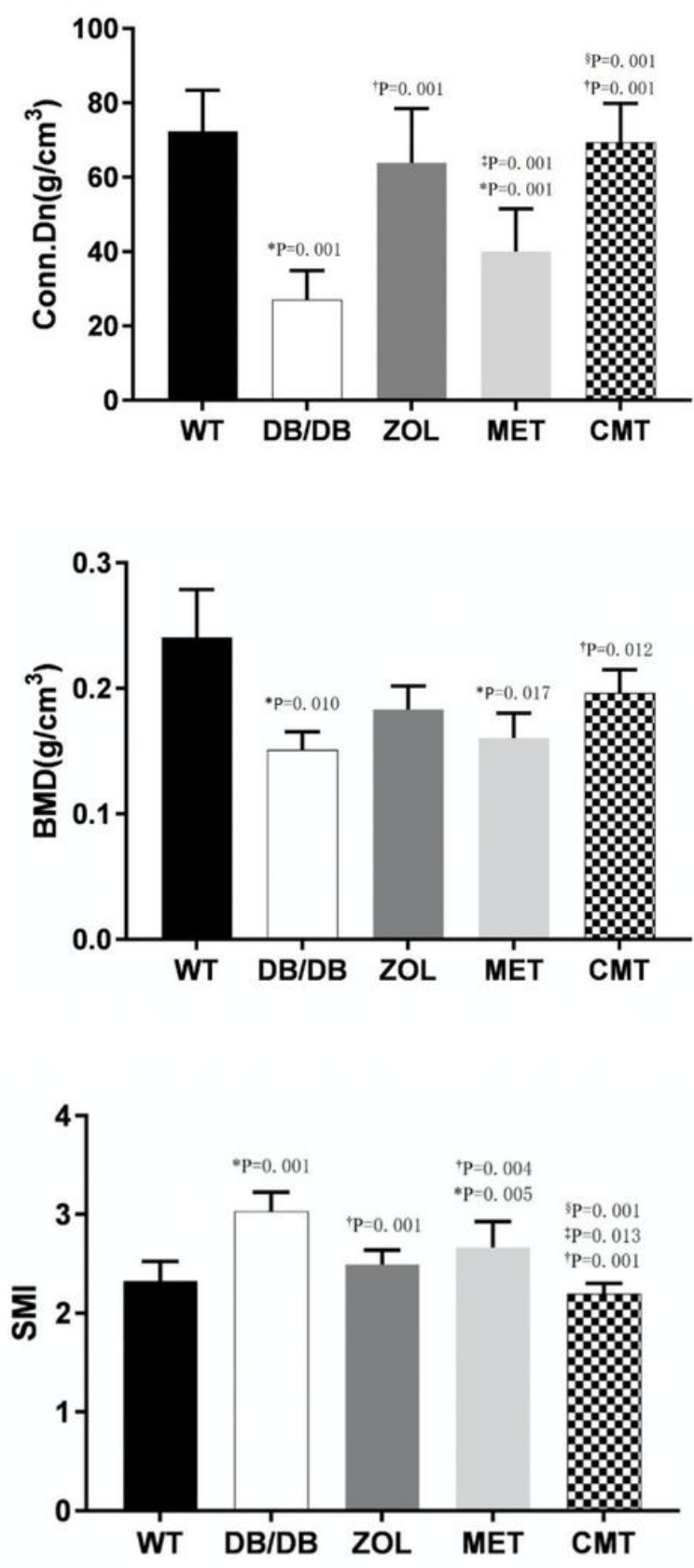

W WT

$\square$ DB/DB

ZOL

MET

$\infty$ CMT

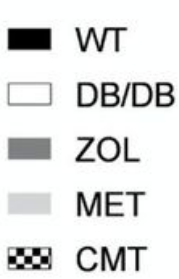

Figure 6

is composed of 7 data graphs scanned by Micro-ct,Data are expressed as mean \pm standard deviation

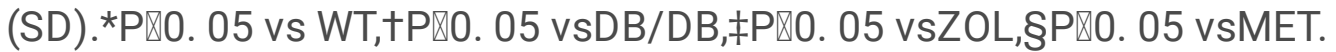

